\title{
Monolitic Hybrid Transmitter-Receiver Lens for Rotary On-Axis Communications
}

\author{
René Kirrbach ${ }^{1,2, *(1)}$, Michael Faulwaßer ${ }^{1}$, Tobias Schneider ${ }^{1}$, Philipp Meißner ${ }^{1}$, \\ Alexander Noack ${ }^{1}$ and Frank Deicke ${ }^{1}$ \\ 1 Department of Wireless Microsystems, Fraunhofer IPMS, 01109 Dresden, Germany; \\ michael.faulwasser@ipms.fraunhofer.de (M.F.); tobias.schneider@ipms.fraunhofer.de (T.S.); \\ philipp.meissner@ipms.fraunhofer.de (P.M.); alexander.noack@ipms.fraunhofer.de (A.N.); \\ frank.deicke@ipms.fraunhofer.de (F.D.) \\ 2 Faculty of Electrical and Computer Engineering, Technical University of Dresden, 01069 Dresden, Germany \\ * Correspondence: rene.kirrbach@ipms.fraunhofer.de or rene.kirrbach@mailbox.tu-dresden.de
}

Received: 29 January 2020; Accepted: 19 February 2020; Published: 24 February 2020

\begin{abstract}
High-speed rotary communication links exhibit high complexity and require challenging assembly tolerances. This article investigates the use of optical wireless communications (OWC) for on-axis rotary communication scenarios. First, OWC is compared with other state-of-the-art technologies. Different realization approaches for bidirectional, full-duplex links are discussed. For the most promising approach, a monolithic hybrid transmitter-receiver lens is designed by ray mapping methodology. Ray tracing simulations are used to study the alignment-depended receiver power level and to determine the effect of optical crosstalk. Over a distance of $12.5 \mathrm{~mm}$, the lens achieves an optical power level at the receiver of $-16.2 \mathrm{dBm}$ to $-8.7 \mathrm{dBm}$ even for misalignments up to $3 \mathrm{~mm}$.
\end{abstract}

Keywords: hybrid lens; optical wireless communications; Li-Fi; freeform lens; optic design; rotary interfaces; rotary joint; wireless rotary electrical interface; rotating electrical connectors; full-duplex data transfer; Gigabit-Ethernet; industrial communications; real-time

\section{Introduction}

Reliable, real-time connectivity is the backbone of industrial automation. Data transmission over rotating parts is required in a broad range of applications such as wind turbines [1], industrial communications [2,3], surveillance radars [3-7], military [2,8], aerospace [9] and many more. Table 1 gives an overview of the most important transmission principles used in rotary communication links. Slip rings were widespread in the past. However, due to mechanical contact they suffer wear and tear which limits their durability $[1,2,5,6,9-11]$ and thus increases maintenance costs. Precious brush materials and lubricants are used to extend lifetime [12] at the expense of increased system complexity and higher costs. Therefore, contactless data transfer is favored nowadays $[6,10]$. Life times of several hundreds of revolutions are common and rotation speeds in the order of magnitude of $10^{3} \mathrm{rpm}$ or even $10^{4} \mathrm{rpm}$ are reached with contactless methods.

Capacitive-based near-field transmission links are known as reliable and cost-effective [11]. However, the system proposed by Doleschel et al. [13] shows that system complexity of modern solutions is clearly not negligible. Data rates of up to several Gbit/s are possible $[11,13]$. Practical systems support conventional Gigabit-Ethernet and industrial protocols like ProfiNET and EtherCAT for instance. Thus, devices with data rates ranging from $500 \mathrm{kbit} / \mathrm{s}$ to $1 \mathrm{Gbit} / \mathrm{s}$ were developed [13]. The maximum transmission distance is in the range of $1 \mathrm{~cm}$. Inductive coupling is mainly employed for power transfer and only rarely used for high-speed data transmission [14,15]. 
Table 1. Common principles for rotary data transmission and their typical state-of-the-art performance. Values should be understood as orders of magnitude rather than exact values. ${ }^{*}$ Higher values are possible but have not been published yet; $\ddagger$ this work is limited to on-axis scenarios.

\begin{tabular}{lcccccc}
\hline Group & Contact & \multicolumn{5}{c}{ Contactless } \\
\hline Type & Slip Ring & Capacitive & RF & Fiber (FORJ) & OWC \\
\hline Ref. & & & & Single & Multi & \\
\hline data rate/Gbit/s & $0.1 \ldots 3$ & $5 \cdot 10^{-4} \ldots>1$ & $0.054 \ldots 5$ & $10 \ldots 40$ & $>40$ & $>10^{*}$ \\
max speed / rpm & $10^{1} \ldots 10^{4}$ & $10^{3} \ldots 10^{4}$ & $10^{3} \ldots 10^{4}$ & $10^{3} \ldots \cdot 10^{4}$ & $10^{2} \ldots 10^{3}$ & $>1400^{*}$ \\
max revolutions & $10^{7} \ldots 2 \cdot 10^{8}$ & $>10^{8}$ & $>10^{8}$ & $>10^{8}$ & $>10^{8}$ & $>10^{8}$ \\
cost (initial) & medium & low $/$ medium & low $/$ medium & high & very high & low \\
cost (maintenance) & high & low & low & low & high & low \\
RF immunity & weak & medium & weak & strong & strong & strong \\
on-/off axis & off-axis & off-axis & off-axis & on-axis & on-axis & on-axis $\ddagger$ \\
\hline
\end{tabular}

Several systems using conventional low-power radio-frequency (RF) technologies were proposed $[1,10]$ with data rates in the range of tens of Mbit/s or below. Standards like 802.11ac and 802.11ad might be able to provide data rates in the range of Gbit/s. Their main problem is reliability and robustness in terms of ensuring a bandwidth and low-latency data transfer $[2,3,13]$. Future millimeter-wave based communication [20] standards like IEEE 802.11ay might even reach data rates in the range of several Gbit/s to several tens of Gbit/s [11]. However, their practicality and cost-effectiveness has to be proven.

Highest data rates are reached with fiber optical rotary joints (FORJ). Besides their superior data rate in the range of Gbit/s up to multiple tens of Gbit/s per channel $[6,7,13]$, these links provide immunity against RF. Single-fiber systems only consist of an optical transceiver at both sides and optical fibers in-between. However, due to sophisticated mechanical alignment [8], these systems are expensive. Multi-fiber links offer even higher data rates but exhibit a very high complexity [6].

Optical wireless communications (OWC) aim to combine the advantages of rotary FORJ with relaxed mechanical tolerances, reduced system complexity and thus lower costs. Initially only light emitting diode (LED) based systems with data rates in the lower Mbit/s range [2,3] or laser diode based uni-directional links were demonstrated [21]. Faulwaßer et al. [19] introduced a full-duplex link for data rates of up to $10 \mathrm{Gbit} / \mathrm{s}$ for on-axis rotary data transfer. Similar to fiber-based communications, data rates are likely to increase in the future. The demonstrated rotation speed from $0 \mathrm{rpm}$ to $1400 \mathrm{rpm}$ [19] was limited by the test equipment. There is no OWC-exclusive factor limiting the speed. The lifetime of OWC links is expected to be similar to other contactless methods, since there are no significant aging effects. The optoelectronic components, i.e., light emitting diodes (LEDs), laser diodes (LDs) and photodiodes (PDs) are known for high reliability and long lifetime [22-24]. The key element of the transceiver in [19] is a monolithic hybrid lens that acts as transmitter (TX) and receiver (RX) optics in parallel and thereby relaxes the mechanical alignment to several millimeters. The form factor of the transceiver is only $5 \mathrm{~mm} \times 5 \mathrm{~mm} \times 5 \mathrm{~mm}$ [19].

This article investigates the potential of OWC for bidirectional, full-duplex, on-axis rotary scenarios and describes how to design a monolithic, hybrid TX-RX lens. In Section 2 a channel model is used to derive some adequate figures of merit. The use of a hybrid lens is motivated by discussing several realization approaches of rotary OWC. Next, the design procedure of a hybrid lens is described and the choice of design parameters is discussed. In Section 3, the performance of the lens and a second system is evaluated and compared using optical ray tracing simulations. Thereby, the alignment-depended optical signal power at the receiver and optical crosstalk is studied. The results are discussed in Section 4 . Finally, Section 5 provides a short summary. 


\section{Materials and Methods}

\subsection{Fundamental Concepts}

\subsubsection{Channel Model}

OWC use optical emitters like LEDs or LDs at the TX to convert an electrical signal into the optical domain. A PD is used for back-conversion at the RX. Similar to FORJ, both transceivers are placed in front of each other [25]. OWC use lenses instead of optical fibers to enable larger mechanical tolerances. The key goal for the designer is to increase the optical power $P_{\mathrm{PD}}$ that falls onto the PD in order to improve the RX signal-to-noise ratio (SNR) [26] and to minimize the bit-error-rate (BER). If the SNR is already sufficient, the excess can be converted into a higher data rate by increasing bandwidth or applying a multilevel modulation scheme.

There will always be a misalignment between both transceivers due to positioning, assembly tolerances or vibrations. The rotation axis might even exhibit a nutation, i.e., nonideal motion around the ideal rotation axis. The combination of these nonidealities and the communication distance leads to a minimum field of view (FOV) that is required for robust operation. The FOV sizes are denoted by the half-opening angles $\theta_{\mathrm{TX} \text { FOV }}$ and $\theta_{\mathrm{RX}}$ FOV. In order to ensure eye-safe operation, i.e., to classify the system as laser class 1 according to IEC 60825-1:2014 (DIN EN 60825-1:2015-07) [27], the optical transmitter power $P_{\mathrm{TX}}$ is limited. Therefore, efficient transceiver design is required to meet link-budget requirements, ensure eye-safety and to maximize data rate. The designer tries to maximize the dynamic range by keeping the TX and RX performance constant within a plane perpendicular to the optical axis [28]. In other words, TX has to provide constant irradiance $E_{\mathrm{TX}}$ and RX has to detect the same signal level within this plane.

For a moment we assume the distance $z$ between both transceivers is large compared to their apertures. Although this does not fully apply for short distances, the assumption is useful to show the fundamental dependencies. As a result, we can assume that parallel rays are incident onto RX. For a homogeneous FOV, the optical power $P_{\mathrm{PD}}$ can be expressed as product of the irradiance $E_{\mathrm{TX}}(z)$, the effective receiver input aperture $A_{\mathrm{RX} \text {,eff }}$ and the geometrical coupling coefficient $\epsilon_{\mathrm{c}}$ as shown in Equation (1). For the ideal optical link, TX and RX have the same FOV size and both FOVs are placed on the optical axis. In practice, both FOVs may differ or they might be misaligned. The geometrical coupling coefficient $\epsilon_{\mathrm{c}}$ quantifies this misalignment. It becomes crucial for short-distance communication as we know from the challenging assembly tolerances of FORJ. $\epsilon_{\mathrm{c}}$ is defined as the fraction of the illuminated area of TX, which is overlapping with the FOV of the RX divided by the total illuminated area $A_{\mathrm{FOV}}$ TX. For the ideal optical channel, i.e., equally sized TX and RX FOVs with no misalignment, $\epsilon_{\mathrm{c}}=1$ applies.

$$
P_{\mathrm{PD}}=E_{\mathrm{TX}}\left(z, \theta_{\mathrm{TX} \mathrm{FOV}}\right) \cdot A_{\mathrm{RX}, \mathrm{eff}}\left(\theta_{\mathrm{RX} \mathrm{FOV}}\right) \cdot \epsilon_{\mathrm{C}}
$$

The effective receiver input aperture $A_{\mathrm{RX}, \mathrm{eff}}\left(\theta_{\mathrm{RX}} \mathrm{FOV}\right)$ is expressed as product of active PD area $A_{\mathrm{PD}}$, optical gain $g\left(\theta_{\mathrm{RX} F O V}\right)$ and efficiency $\eta_{\mathrm{RX}}\left(\theta_{\mathrm{i}}\right)$ at the angle of incidence $\theta_{\mathrm{i}}$ as it is shown in Equation (2). A large PD area $A_{\mathrm{PD}}$ is favorable for the link budget but goes along with a large PD capacitance, which limits the modulation bandwidth (BW) [26,29]. Consequently, a PD with large $A_{\mathrm{PD}}$ but sufficient BW is chosen.

$$
P_{\mathrm{PD}}=E_{\mathrm{TX}}\left(z, \theta_{\mathrm{TX} \mathrm{FOV}}\right) \cdot A_{\mathrm{PD}} \cdot g\left(\theta_{\mathrm{RX} \mathrm{FOV}}\right) \cdot \eta_{\mathrm{RX}}\left(\theta_{\mathrm{i}}\right) \cdot \epsilon_{\mathrm{C}}
$$

The RX FOV should always be chosen as small as possible, since the optical gain $g\left(\theta_{\mathrm{RX}}\right.$ FOV $)$ decreases with increasing FOV due to conservation of Ètendue [30]. Moreover, a restricted RX FOV improves the robustness against noise and interchannel interference. Next, $g\left(\theta_{\mathrm{RX}}\right.$ FOV $)$ is substituted by the maximum theoretical optical gain [30] as shown in Equation (3). Now $\eta_{\mathrm{RX}}\left(\theta_{\mathrm{i}}\right)$ is used as a figure of merit for the optical RX efficiency. The ideal lossless receiver achieves $\eta_{R X}\left(\theta_{i}\right)=1$ for all $\theta_{\mathrm{i}} \in\left[0, \theta_{\mathrm{RX}} \mathrm{FOV}\right]$. The angle $\theta_{\mathrm{RX}, \mathrm{PD} \text {,max }}$ denotes the maximum coupling angle from the RX optics to the 
PD surface normal. In the next step, the irradiance $E_{\mathrm{TX}}\left(z, \theta_{\mathrm{TX}}\right.$ FOV $)$ is substituted by the product of the optical TX power $P_{\mathrm{TX}}$ and the TX efficiency $\eta_{\mathrm{TX}}$ divided by the illuminated spot area $A_{\mathrm{FOV}} \mathrm{TX}$.

$$
P_{\mathrm{PD}}=\frac{P_{\mathrm{TX}} \cdot \eta_{\mathrm{TX}}}{A_{\mathrm{FOV} \mathrm{TX}}} \cdot\left(\frac{n_{1} \cdot \sin \theta_{\mathrm{RX}, \mathrm{PD}}}{n_{\text {air }} \cdot \sin \theta_{\mathrm{RX} \mathrm{FOV}}}\right)^{2} \cdot A_{\mathrm{PD}} \cdot \eta_{\mathrm{RX}}\left(\theta_{\mathrm{i}}\right) \cdot \epsilon_{\mathrm{c}}
$$

$A_{\mathrm{FOV}} \mathrm{TX}$ is replaced by the corresponding triangular relationship, which includes the

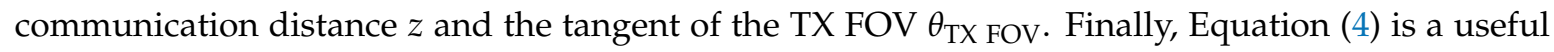
expression for the most important geometric dependencies of $P_{\mathrm{PD}}$.

$$
P_{\mathrm{PD}}=\frac{P_{\mathrm{TX}} \cdot \eta_{\mathrm{TX}}}{\pi \cdot\left(z \cdot \tan \theta_{\mathrm{TX} \mathrm{FOV}}\right)^{2}} \cdot\left(\frac{n_{1}}{\sin \theta_{\mathrm{RX} \mathrm{FOV}}}\right)^{2} \cdot A_{\mathrm{PD}} \cdot \eta_{\mathrm{RX}} \cdot \epsilon_{\mathrm{C}}
$$

The performance merits from Equation (4) are $\eta_{\mathrm{TX}}, \eta_{\mathrm{RX}}$ and $\epsilon_{\mathrm{c}}$. The transmitter efficiency $\eta_{\mathrm{TX}}$ specifies how much of the emitted power $P_{\mathrm{TX}}$ is concentrated into the FOV. Its loss mechanisms are $\zeta_{\mathrm{TX} M}$ and $\zeta_{\mathrm{TX} F}$. $\zeta_{\mathrm{TX} M}$ describes rays that strike the target plane outside the FOV. $\zeta_{\mathrm{TX} F}$ describes back-reflected rays due to Fresnel-reflections.

Since $\eta_{T X}$ contains no information concerning irradiance homogeneity, we additionally introduce the effective transmitter efficiency $\eta_{\mathrm{TX}}$ eff. It is defined according to Equation (5) by using the minimum irradiance within the FOV $E_{\min }[28,31]$. The difference between $\eta_{\mathrm{TX}}$ and $\eta_{\mathrm{TX}}$ eff is called the inhomogeneity factor $\zeta_{\mathrm{TX} H}$.

$$
\eta_{\mathrm{TX} \text { eff }}=\frac{E_{\mathrm{min}}}{E_{\mathrm{min}, \text { ideal }}}=\frac{E_{\mathrm{min}} A_{\mathrm{FOV} \mathrm{TX}}}{P_{\mathrm{TX}}}
$$

The sensitivity of RX is typically limited by the interaction of several internal noise mechanisms that exhibit a Gaussian probability function [26]. The signal can additionally be corrupted by optical crosstalk, i.e., adjacent communication channels. This nonGaussian noise has a limited range of variation. This effect introduces a power penalty $P P$ [26] that reduces the usable peak-to-peak amplitude of the signal. The optical power that falls onto the PD consists of a signal part $P_{\mathrm{PD}}$ and another part arising from crosstalk $P_{\mathrm{PD} \text { cross. }}$. The corrected power value $P_{\mathrm{PD}} \mathrm{PP}$ takes the eye-closure effect into account by subtracting the $P_{\mathrm{PD} \text { cross }}$ from $P_{\mathrm{PD}}$ [26]. It holds $P_{\mathrm{PD} P P}<P_{\mathrm{PD}}$ as soon as crosstalk is present. In this work, all optical power values are understood as average values to ensure comparability with literature. When dealing with the power penalty the signal strength is considered in a peak-to-peak manner. It is still valid to consider average values as long as the extinction ratio of the signal and the crosstalk signal is equal. This assumption applies to our case, since both link directions are designed equally and the crosstalk arises from the signal itself.

\subsubsection{Ideal Arrangement}

The ideal arrangement consists of TX and RX placed at the same position on the optical axis as shown in Figure 1. Both FOVs are equal in size and they fully overlap. The arrangement can be realized by using an LED. On the one hand, applying a forward-bias to the LED causes a forward-current and leads to photon generation. Biasing the PN-junction reversely enables fast photo-detection on the other hand. As a result, the transceiver only needs a single optoelectronic component for TX and RX. Data rates of up to $150 \mathrm{Mbit} / \mathrm{s}$ were demonstrated for close distances [32]. However, the LED is a rather bad PD. It has a low responsivity, small area $A_{\mathrm{PD}}$ and low bandwidth [32]. Although a bidirectional link is realizable, data transfer is restricted to half-duplex mode, because the link direction is switched in time domain. 


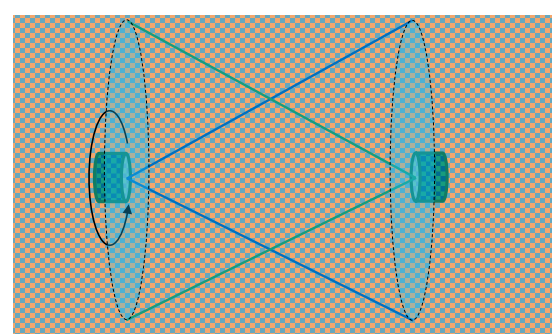

Figure 1. Ideal rotary arrangement: transmitter (TX) (blue) and receiver (RX) (green) of a transceiver are placed on the optical axis at the same position. The field of view (FOV) of TX and RX overlap ideally.

For high-speed bidirectional data transfer in full-duplex mode, TX and RX are separated. The optical arrangement becomes more challenging and design trade-offs have to be met. The following section introduces several geometrical arrangements for optical wireless rotary communication scenarios and compares their performance.

\subsubsection{Geometrical Arrangements}

Figure 2 shows three geometrical approaches for rotary OWC. In Figure 2a TX and RX are placed next to each other separated by a spacing $d$. In Figure $2 b$, TX and RX are radially separated regarding the optical axis. Third, both elements arranged along the optical axis and the front element are transparent as illustrated in Figure 2c.

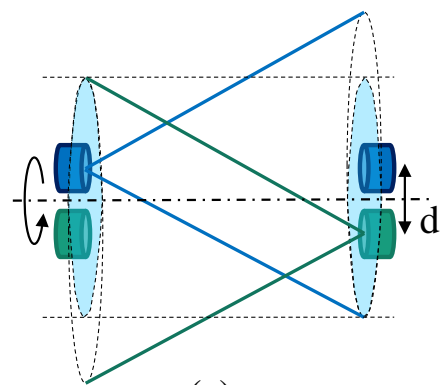

(a)

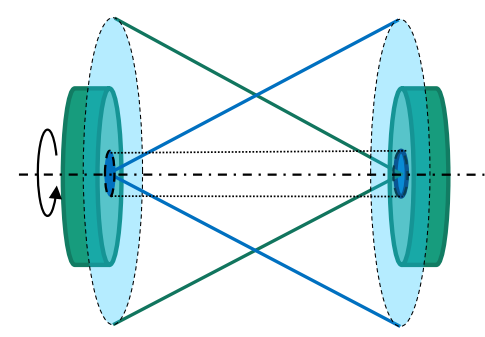

(b)

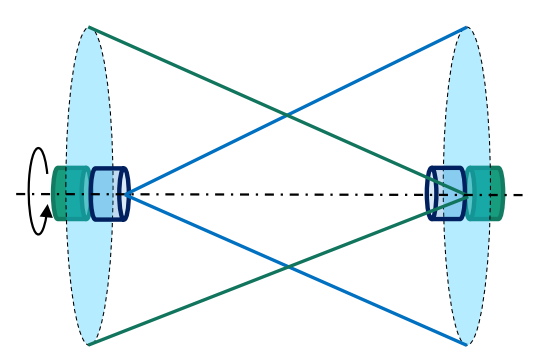

(c)

Figure 2. Schematic illustration of arrangements of separated TX (blue) and RX (green). (a) TX and RX are placed next to the rotation axis. The FOV of TX and RX overlap only partially. (b) TX and RX are placed at the optical axis and they are separated in radial direction. (c) TX and RX are placed in a line along the optical axis and the front element is transparent.

Approach (a) is used by commercial low data rate IrDA transceivers like Vishays TFBS4711 [33] but also by a high-speed transceiver demonstrated by Faulwaßer et al. [34]. The axis of rotation is placed trough one of the elements or between them. This leads to misaligned FOVs. The designer tries to minimize the spacing $d$. Next, both FOVs are chosen large enough to ensure a sufficient $\epsilon_{\mathrm{c}}$. As we learned from Equation (4), $P_{\mathrm{PD}}$ scales with $1 / \tan \theta_{\mathrm{TX} \text { FOV }}^{2}$ and $1 / \sin \theta_{\mathrm{RX} \text { FOV }}^{2}$. This penalty is typically significant. A numerical example is given in Chapter 3.

This penalty is not present for the radial separation from Figure $2 b$. The approach directly provides aligned FOVs and a high $\epsilon_{\mathrm{c}}$. TX can be placed in the center surrounded by RX as shown in Figure $2 b$ or vice-versa.

The approach shown in Figure $2 \mathrm{c}$ avoids shadowing of the back component by designing the front element transparent. If the front component is TX, it has to have separated emission and absorption bands, i.e., it must exhibit a Stokes shift similar to the fluorescence materials [35]. The issue here is the back plane of the emitter: on the one hand, it has to be reflective to direct the transmitted signal towards the other transceiver. On the other hand, it has to be transparent for the incoming signal. This contradiction does not seem to be easily resolved. 
Clearly the radial separation from Figure $2 b$ has superior performance over Figure $2 a$ and has no fundamental concept issue like architecture Figure 2c. However, what is the best way of realizing radial separation? Figure 3 illustrates three principles.

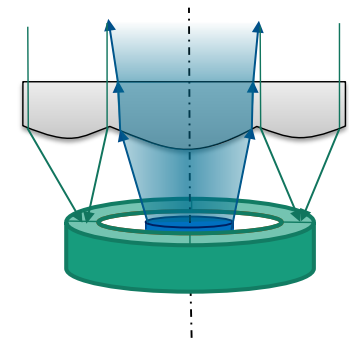

(a)

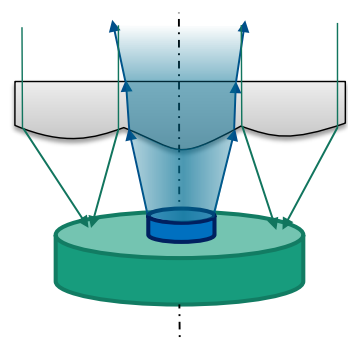

(b)

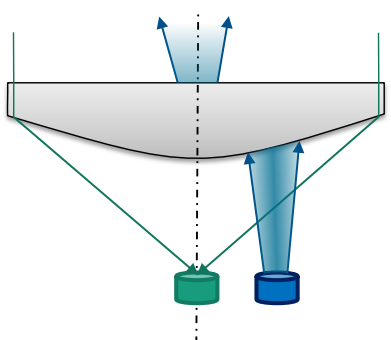

(c)

Figure 3. Schematic illustration of different radial separations of TX (blue) and RX (green) with an optical element (grey). (a) Direct integration of TX and RX into a plane. (b) Stacking an emitter chip onto a PD. (c) An optical element is used to homogenize the TX ray bundle and move it to the optical axis.

Designing a transmitter element surrounded by one or more high-speed PDs or vice versa on a single chip, like it is shown in Figure 3a, comes with many design challenges including process compatibility. Thereby, it seems easier to produce the chips separately and stack them afterwards. A small emitter die is bonded onto a large area PD chip as it is depicted in Figure 3b. The emitter is contacted with bonds or directly trough the PD chip. The PD could be separated into multiple parts to ease the contacting and to reduce the transit time of the electrons and holes as they might limit the bandwidth [26]. The main issues of this approach include crosstalk between TX and RX, shadowing of the PD by the emitter and the fact that both chips are custom designs.

By using an optical system like it is shown in Figure 3c, conventional emitter and receiver chips can be used. Those dies are placed next to each other and a hybrid TX-RX lens is used to redirect the rays to achieve radial separation. The spatial separation of both chips is favorable to reduce electrical crosstalk. Injection molding allows the fabrication of the lens in high volume [36] and low cost. Ultra-precise drilling and milling [36] is used to produce the mold tool.

Since a part of the lens is used to direct the TX rays, the maximum theoretical gain $g_{\text {max }}$ cannot be reached. From the PDs point of view, the solid angle of the TX lens part is not used for optical concentration. In Equation (4) this is expressed by a reduced $\eta_{\mathrm{RX}}$.

In summary, using a hybrid lens for radial separation is most promising: besides the potential for $\epsilon_{\mathrm{c}} \approx 1$, commercial emitter and PD chips can be used. Since the hybrid lens can be fabricated at low cost by injection molding, the system costs are expected to be low.

\subsection{Hybrid Lens}

\subsubsection{Concept}

In order to achieve separation in radial direction, the lens is divided into a TX and RX part, i.e., a center part and a surrounding one. Generally, both parts consist of nonrotationally freeform surfaces at the top and bottom of the lens to form a constant irradiance pattern $E_{\mathrm{TX}}$ and provide a homogeneous gain $g$. In order to limit the lens thickness $t$, Fresnel-structures could be applied to the top and bottom surface. However, it is favorable to keep the top flat to improve reliability, since cavities tend to fill up with particles.

There are two possible arrangements of TX and RX:

1. The emitter is placed centrally and the PD is positioned off-axis as depicted in Figure 4a. In this case only the emission profile of the emitter has to be adjusted. This includes the homogenization of the profile and an adjustment of the angle $\theta_{\mathrm{TX}}$. This can ideally be achieved with a single 
freeform interface. Therefore, the lower surface can be used for beam shaping and the top surface can be flat. The downside of this approach is a challenging RX lens design: the focus point of the $\mathrm{RX}$ lens part is off-axis.

2. The PD is placed centrally and the emitter is positioned off-axis as shown in Figure $4 \mathrm{~b}$. The TX lens part fulfills two tasks: first, it compensates the displacement of the ray bundle regarding the optical axis. Second, it reshapes the ray bundle to the anticipated FOV. Because two surfaces are required, the top aperture of the lens cannot be flat. On the other hand, the design of the RX lens part is simplified, because the focal point is on the optical axis. If the TX part is neglected, the RX lens can be designed to be rotationally symmetrical. However, the shadowing effect of the TX part introduces a nonrotationally symmetric factor. Theoretically, this can be partly compensated by a nonrotationally symmetric $\mathrm{RX}$ lens part. The shadowing effect is also mitigated by minimizing the size of TX.

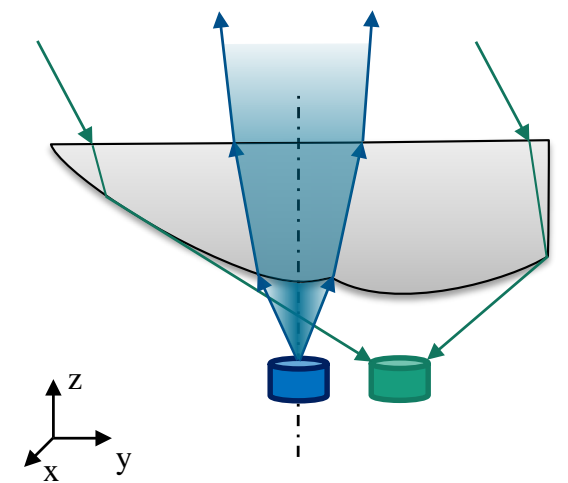

(a)

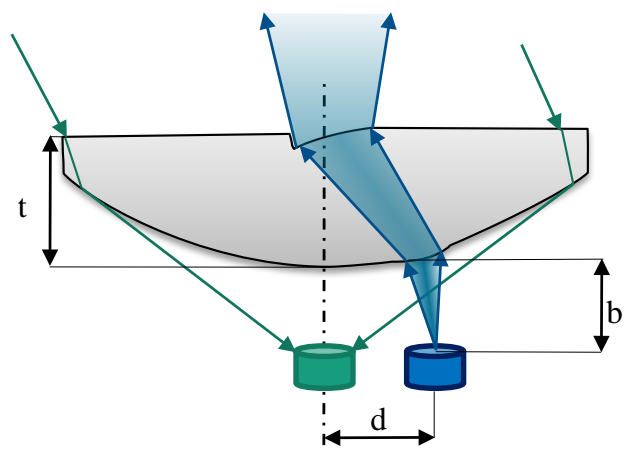

(b)

Figure 4. Ray path in the hybrid lens system. (a) Center TX (blue) and off-axis RX (green). (b) Center RX and off-axis TX.

We choose Approach 2, due to the simplified RX lens design. In this configuration, the lens thickness $t$ typically results from the vertical distance between both TX surfaces. A low $t$ is desirable for size, weight and cost reduction. The costs are reduced, because less material is needed and due to faster processing [36,37]. Nevertheless, a certain thickness $t$ is required to keep the refraction angles at the TX surfaces low in order to limit undesirable Fresnel-reflections. If the RX part determines $t$, the surface can be split into several Fresnel-grooves to reduce $t$.

\subsubsection{Optic Design Methods}

The hybrid lens is a nonimaging optical system. It can be designed by two fundamental approaches [38]: numerical optimization and direct calculation.

Numerical optimization is a straight-forward approach for designing complex optic modules. Modern optic simulation tools like Optic Studio Zemax enable forming and optically simulating arbitrarily shaped optics by overlapping parametric objects. Optimization algorithms like the Levenberg-Marquardt algorithm are used for adjusting parameters of those objects until a sufficiently good result is achieved. Due to the large amount of variables, the optimization is typically inefficient, because of many local minima in the merit function [38]. As a result, it is easy to find a solution, but its performance is very limited, especially if the systems become more complex.

In contrast, direct calculation algorithms follow well-defined design procedures and yield deterministic outcomes. Thereby, they provide better results than numerical optimization methods [38], especially if the systems are complex. A great variety of design methods are known, for example ray mapping [39-46], forming surfaces using Cartesian ovals [47], the simultaneous multiple surface method in 2D [48] and 3D [38,49] or the tailored freeform design method proposed by Ries and Muschaweck [50]. Nowadays ray mapping approach, i.e., the combination of energy mapping in 
conjunction with geometrical surface construction, is in the focus of illumination research [39-46]. Here, a mass-transfer problem is solved by transforming the source power irradiance $E_{\mathrm{s}}$ into the target power irradiance $E_{\mathrm{t}}$. This transformation is represented by a mapping $\phi: \Omega_{\mathrm{s}} \rightarrow \Omega_{\mathrm{t}}$ from the source domain $\Omega_{\mathrm{s}}$ to the target domain $\Omega_{\mathrm{t}}$. Then, the laws of refraction and reflection are applied for $k \times l$ points to calculate a corresponding vector field $N$ containing the surface normals $\boldsymbol{n}_{i, j}$ with $i \in[1, l], j \in[1, k]$. The challenge is to find a mapping $\phi$ which yields a vector field $N$ that satisfies the integrability condition for a continuous surface. This condition is shown in Equation (6) [41,42,50]. It states that $N$ has to be curl-free or exhibit at least minimum curl.

$$
N \cdot(\nabla \times N)=0
$$

Nowadays, parametrization and consecutive optimization are widely employed for generating a mapping $\phi[28,31,39,40]$. Circular shaped FOV are formed with the mapping shown in Figure $5 \mathrm{a}$. An equi-flux grid around the source in spherical coordinates $\theta_{\mathrm{s}}, \phi$ is mapped onto a target grid in polar coordinates $\beta, r$. In 3D, mappings like these typically lead to a normal vector field $N$ with substantial curl. Therefore, an optimization procedure purposely distorts the target grid, for instance by varying $r$, to improve the performance. It was shown that this approach works well for on-axis scenarios $[28,39]$. In case of an off-axis placed emitter and two optical surfaces, the scenario is more challenging due to the nonparaxial nature. Hence, the result will deviate from the anticipated irradiance pattern. An additional variable $\beta$ could be used in lateral direction as illustrated in Figure 5a. The downside is a slower optimization process.

(a)

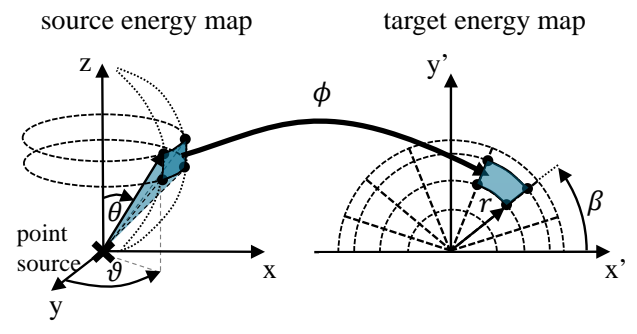

(b)

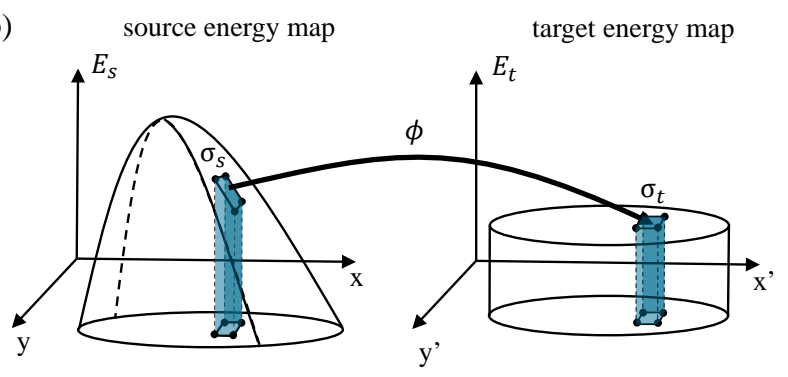

(c)

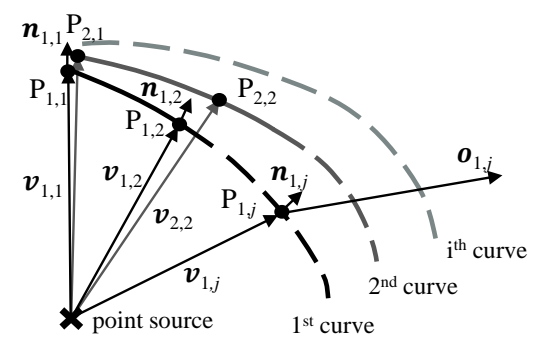

Figure 5. Energy and ray mapping. (a) Equi-flux grids at the source and target. Adapted from Wang et al. [39]. (b) Mapping from non equi-flux source to anticipated target irradiance. (c) Input vectors $v_{i, j}$ and output $\boldsymbol{o}_{i, j}$ vectors are used to construct the surface geometry by subsequently calculating points $P_{i, j}$ and their normals $\boldsymbol{n}_{i, j}$.

For the TX lens part of the hybrid lens, inefficient optimization can be avoided by taking the curl of $\phi$ directly into account. An initial curl-free mapping $\phi_{0}$ is generated, assuming that the resulting vector field $N$ exhibits minimum curl [42]. Figure $5 \mathrm{~b}$ illustrates the irradiance in front of the source $E_{\mathrm{s}}$ and the anticipated irradiance at the target plane $E_{\mathrm{t}}$. The mapping $\phi$ has to ensure that the infinitesimal area elements at $\sigma_{\mathrm{s}}$ and $\sigma_{\mathrm{t}}$ are passed by the same flux. This is achieved by expansion or contraction of the area elements. Mathematically spoken, the mapping has to satisfy Equation (7) for every $\sigma_{\mathrm{s}}$ of the source grid $[42,46]$.

$$
\operatorname{det}\left(\nabla \phi\left(\sigma_{\mathrm{s}}\right)\right) \rho_{\mathrm{t}}\left(\phi\left(\sigma_{\mathrm{s}}\right)\right)=\rho_{\mathrm{s}}\left(\sigma_{\mathrm{s}}\right)
$$


The term $\operatorname{det}(\nabla \phi(\sigma))$ represents the expansion and contraction of the area element [46]. Although many mappings $\phi$ might satisfy Equation (7), there is only a single one that minimizes transport cost [46]. Solving Equation (7) turns out to be nontrivial [42,46]. In this article, the algorithm proposed by Wu et al. [46] is used since it provides good convergence.

For the RX lens part, a rotationally symmetric concentrator lens is designed. It consists of two sections: the center is based on refraction, whereas the outer section works with total internal reflection (TIR). TIR is generally superior over refraction for large angles $\theta_{R X}$ PD i since it limits the lens diameter and reduces Fresnel-losses [51]. Similar to the TX lens part, the surface is calculated from input and output vectors $v_{i, j}$ and $\boldsymbol{o}_{i, j}$. In the first attempt, the edge ray principle [30] was applied for generating the input vectors $v_{i, j}$. Due to the TX lens part and the discontinuity between both RX lens sections, the edge ray principle is not valid. Therefore, the gain will vary over the FOV and may drop at certain alignments. In the second attempt, this issue is addressed by defining the input vectors for a range of angles of incidence rather than only for the maximum incidence angle. The output rays $\boldsymbol{o}_{i, j}$ are derived from coupling angles $\theta_{\mathrm{RX}} \mathrm{PD}$ i to the PD active area. Ideally, they cover the whole half-space in front of the PD.

The normal vector fields $\mathbf{N}$ of both lens parts and an initial point $\mathbf{P}_{1,1}$ for each surface is used to calculate a finite number of surface points $\mathbf{P}_{i, j}$. The result is only a point cloud representation for the optical surfaces. Nonuniform rational B-splines (NURBS) [52] are used for interpolation. NURBS are very flexible and they are supported in the most popular computer aided design formats, which makes them suitable for optical simulation in third-party software and for subsequent fabrication.

\subsection{System with Separated $T X$ and $R X$}

In order to show the potential of the methodology and the concept of the hybrid TX-RX lens, a second optical wireless link based on the principle shown in Figure 2a is developed. A concave lens is chosen for TX to widen up the beam. A convex RX lens is used for optical concentration. The distance $d$ between the TX and RX determines the maximum size of both lenses. The RX lens must not be too small to enable a sufficient optical gain $g$. Therefore, $d$ will be larger compared to the hybrid lens approach. The exact optical parameters are determined by numerical optimization. The hybrid TX-RX lens and the fully separated TX-RX lens system are denoted by H TX-RX and S TX-RX respectively.

\subsection{Simulation Parameters}

For optical simulations, Monte Carlo ray tracing in Optic Studio Zemax 17 is used. For each simulation, $5 \cdot 10^{5}$ rays are traced. Thereby, polycarbonate lenses are used with a refractive index of $n_{1}=1.57$ at $\lambda=940 \mathrm{~nm}$. We especially investigate Fresnel-reflections which lead to direct optical crosstalk $P_{\mathrm{PD} \text { cross }}$ if the reflected rays reach the PD. The detector is a circular shaped PIN-PD with a radius of $100 \mu \mathrm{m}$. The $\mathrm{LD}$ has an output power of $P_{\mathrm{TX}}=4 \mathrm{~mW}=6 \mathrm{dBm}$ at $\lambda=940 \mathrm{~nm}$. First, the transmitter part is considered. Then, the full channel is characterized. In order to give a comparable result to other rotary communication technologies, the link distance is set to $12.5 \mathrm{~mm}$.

Conventionally, optical concentrators are characterized by estimating the gain over the angle of incidence by assuming parallel light [53]. However, Figure 6a shows a large divergence of the incident rays. Thus, the assumption of parallel rays does not apply for the present arrangement. The RX performance depends directly on the TX characteristics. Therefore, it is evaluated by the full-channel simulation.

\section{Results}

Figure 6a shows a render of a cross-section through the lens. It is $6.0 \mathrm{~mm}$ in diameter and has a thickness of $t=2.0 \mathrm{~mm}$ in the center and $t=2.7 \mathrm{~mm}$ at the groove. The PD is placed on-axis at the origin. The LD position is $(x, y)=(-0.5 \mathrm{~mm}, 0 \mathrm{~mm})$. 
For the second system, the numerical optimization leads to a separation between LD and PD of $d=1.9 \mathrm{~mm}$. The LD is placed at $x=-0.95 \mathrm{~mm}$ and the PD at $x=0.95 \mathrm{~mm}$. The TX and RX lens have a diameter of $1.3 \mathrm{~mm}$ and $2.2 \mathrm{~mm}$ and a focal length of $-9 \mathrm{~mm}$ and $2.7 \mathrm{~mm}$, respectively.

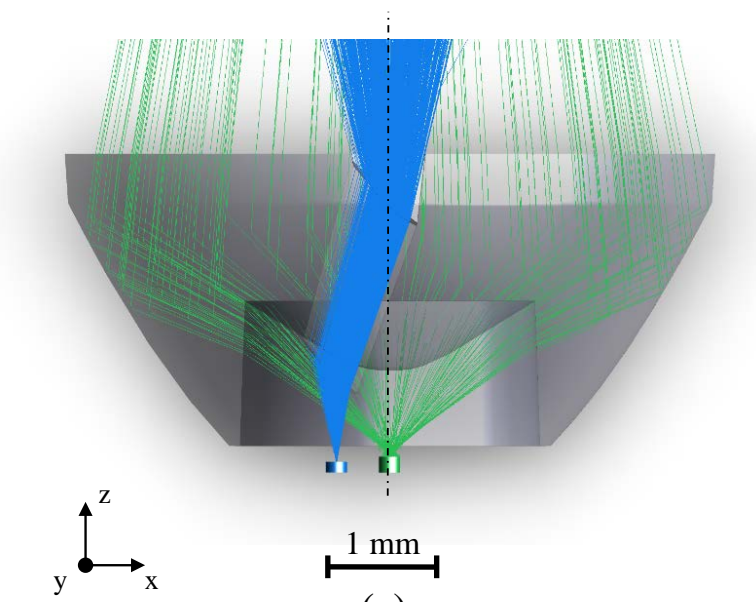

(a)

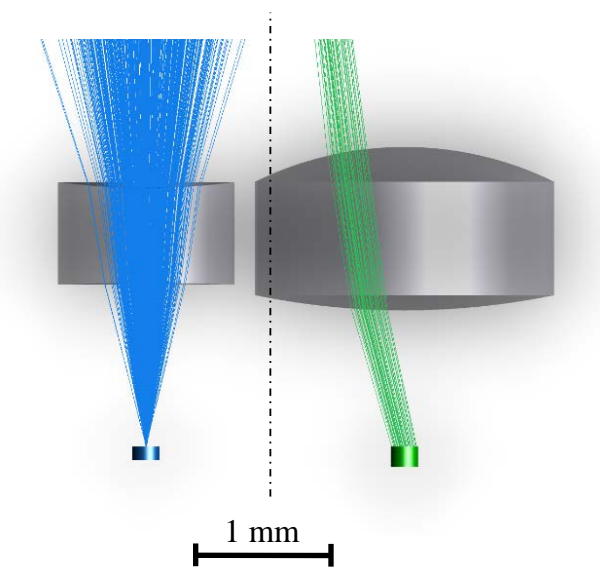

(b)

Figure 6. Render of the cross-section through the optical system with TX rays (blue) and RX rays (green). Both devices are placed on the rotation axis (black dotted line) with no misalignment. Only RX rays that hit the detector are shown. (a) Hybrid TX-RX lens (H TX-RX). (b) Fully separated TX and RX (S TX-RX) in worst-case orientation, i.e., TX lens facing TX lens and RX lens facing RX lens.

\subsection{TX Performance}

Figure 7 shows the irradiance at a distance of $12.5 \mathrm{~mm}$, measured from the TX aperture. Table 2 lists the detailed merits of both profiles. Figure 7a shows the donut-like shaped profile of the LD without optics. The off-axis placement of the LD is directly observable as profile displacement along the $\mathrm{x}$-axis. Figure $7 \mathrm{~b}$ shows that the hybrid TX-RX lens is able to remove the displacement and homogenize the profile. The effective transmitter efficiency $\eta_{T X}$ eff is $57.6 \%$ with a minimum irradiance of $117 \mu \mathrm{W} / \mathrm{mm}^{2}$ at $(x, y)=(1.5 \mathrm{~mm}, 1.5 \mathrm{~mm}) . \eta_{\mathrm{TX}}$ and $\eta_{\mathrm{TX}}$ eff are both reduced by $\zeta_{\mathrm{TX} \mathrm{M}}=24.7 \%$ and $\zeta_{\mathrm{TX} F}=12.1 \%$. Moreover, $\eta_{\mathrm{TX} \text { eff }}$ is lowered by another $\zeta_{\mathrm{TX} H}=5.6 \%$ due to inhomogeneity within the FOV.

Figure 7c shows the irradiance for the system with fully separated TX and RX as described in Section 2.3. Due to the profile displacement, $\zeta_{\mathrm{TX} M}=36.0 \%$ of the power misses the FOV. Moreover, the spherical lenses are not able to correct the donut-shaped profile resulting in a high $\zeta_{\mathrm{TX} \mathrm{H}}=38.6 \%$. As a result, the irradiance is very inhomogeneous within the FOV and it drops down to $25.9 \mu \mathrm{W} / \mathrm{mm}^{2}$ at the right side. This corresponds to $\eta_{\mathrm{TX}}$ eff $=12.8 \%$.

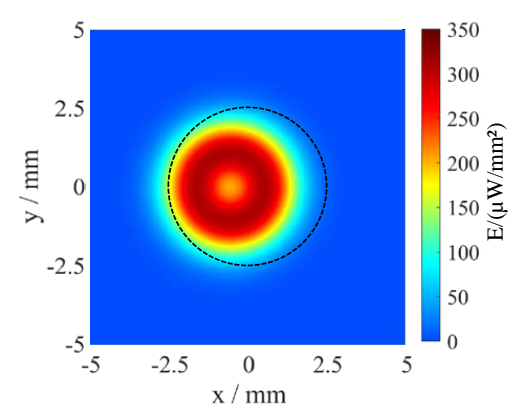

(a)

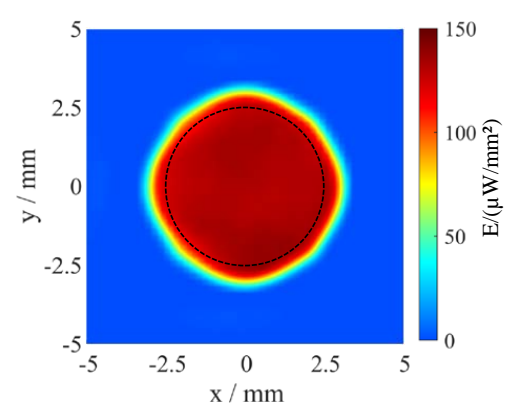

(b)

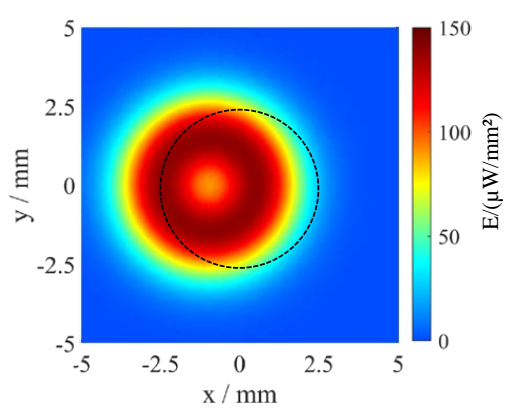

(c)

Figure 7. Irradiance over $z=12.5 \mathrm{~mm}$. (a) For the LD without any optics (LD). (b) For the LD with the hybrid TX-RX lens. (c) For fully separated TX and RX. The black circle highlights the anticipated TX FOV. Note the different color bar scales for the subfigures. 
Table 2. TX merits for the laser diode (LD), the hybrid TX-RX system (H TX-RX) and the separated TX-RX lens system (S TX-RX).

\begin{tabular}{lcccc}
\hline Merit & Unit & LD & H TX-RX & S TX-RX \\
\hline$E_{\min }$ & $\frac{\mu \mathrm{W}}{\mathrm{mm}^{2}}$ & 14.6 & 117 & 25.9 \\
$\eta_{\mathrm{TX}}$ & $\%$ & 89.5 & 63.2 & 51.4 \\
$\eta_{\mathrm{TX} \text { eff }}$ & $\%$ & 7.2 & 57.6 & 12.8 \\
$\zeta_{\mathrm{TX} M}$ & $\%$ & 10.5 & 24.7 & 36.0 \\
$\zeta_{\mathrm{TX}} \mathrm{F}$ & $\%$ & 0 & 12.1 & 12.6 \\
$\zeta_{\mathrm{TX} H}$ & $\%$ & 82.3 & 5.6 & 38.6 \\
\hline
\end{tabular}

\subsection{Full-Channel Performance}

Figure 8a-c displays $P_{\mathrm{PD}}, P_{\mathrm{PD} \text { cross }}$ and $P_{\mathrm{PD} P \mathrm{PP}}$ at a lens-to-lens distance of $12.5 \mathrm{~mm}$ for the hybrid lens. The values are determined by misaligning the receiving transceiver, whereas the transmitting one is placed at the origin. Table 3 shows numerical values for different misalignments in negative $\mathrm{x}$-direction. Figure 8a shows a rotationally symmetric performance in its fundamental structure. However, $P_{\mathrm{PD}}$ is not fully homogeneous within the FOV for a revolution. The largest variation $\Delta P_{\mathrm{PD}}$ over one revolution is reached if the misalignment is between $2 \mathrm{~mm}$ and $3 \mathrm{~mm}$. There, $\Delta P_{\mathrm{PD}}$ is in the range of $3 \mathrm{~dB}$ to $3.29 \mathrm{~dB}$. The crosstalk power $P_{\mathrm{PD} \text { cross }}$ in Figure $8 \mathrm{~b}$ is similarly distributed as $P_{\mathrm{PD}}$ with a power level which is about $10 \mathrm{~dB}$ lower than $P_{\mathrm{PD}}$. As a result, the optical power with applied power penalty $P_{\mathrm{PD} P \mathrm{PP}}$ in Figure $8 \mathrm{c}$ is similar to $P_{\mathrm{PD}}$. This can also be observed in Table 3: the difference between $P_{\mathrm{PD}}$ and $P_{\mathrm{PD}}$ PP is in the range of $0.1 \mathrm{~dB} \ldots 0.9 \mathrm{~dB}$ (1.023...1.230). The crosstalk $P_{\mathrm{PD} \text { cross }}$ consists of two parts: a constant part $P_{\mathrm{PD}}$ cross 1 of $-32.4 \mathrm{dBm}$ and an alignment-depended part $P_{\mathrm{PD}}$ cross 2 .

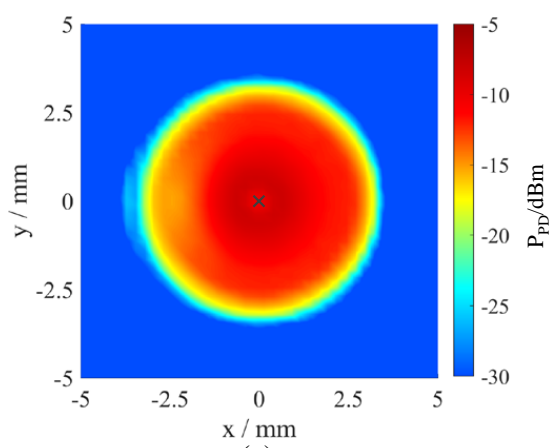

(a)

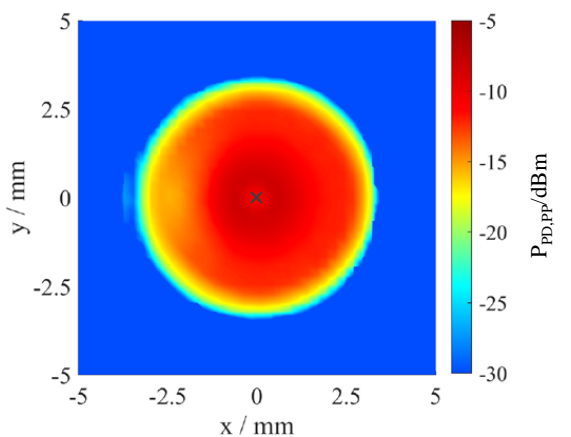

(c)

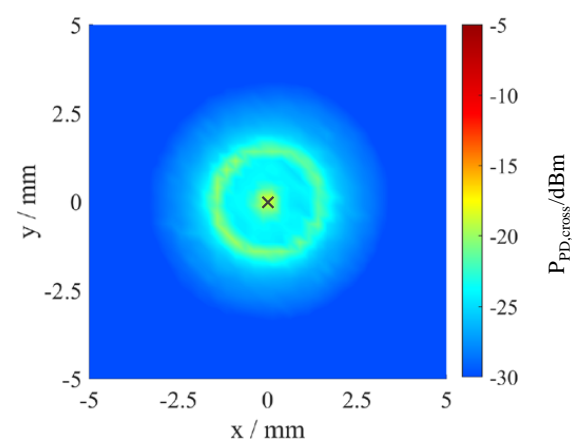

(b)

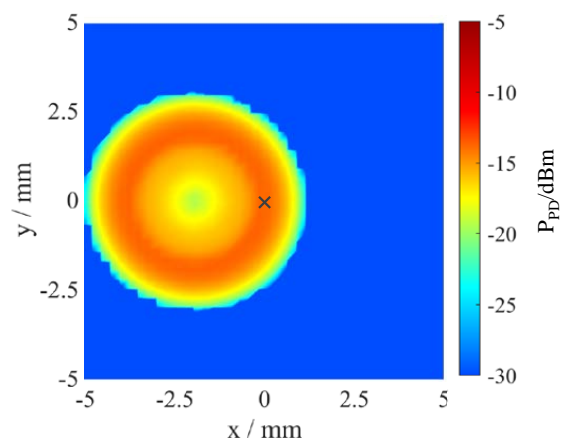

(d)

Figure 8. Incident power $P_{\mathrm{PD}}$ and $P_{\mathrm{PD} \text { cross }}$ over misalignment at $z=12.5 \mathrm{~mm}$. The " $\mathrm{x}$ " marks the axis of rotation. (a) $P_{\mathrm{PD}} / \mathrm{dBm}$ for the hybrid lens. (b) $P_{\mathrm{PD} \text { cross }} / \mathrm{dBm}$ for the hybrid lens. (c) $P_{\mathrm{PD}} \mathrm{PP} / \mathrm{dBm}$ for the hybrid lens. (d) $P_{\mathrm{PD}} / \mathrm{dBm}$ for the system with fully separated TX and RX (Section 2.3) in worst-case orientation, i.e., TX lens facing TX lens and RX lens facing RX lens. Note: The graphs are clipped below $-30 \mathrm{dBm}$ to highlight features within the FOV. Therefore, $P_{\mathrm{PD}}$ cross 1 cannot be seen in Figure $8 \mathrm{~b}$. 
The system with fully separated TX and RX is shown in Figure 8d. It suffers from a displacement of the communication-area in $x$-direction. The effect can be seen in Table 3; a communication is only possible for a misalignment of $0.5 \mathrm{~mm} . .1 \mathrm{~mm}$, depending on the data rate.

Table 3. Minimum $P_{\mathrm{PD}}$ depending on the misalignment in negative $x$-direction $(y=0)$. Values are determined by choosing the minimum value $P_{\mathrm{PD}}$ on a circle around the center with a radius of the misalignment. Values lower than $40 \mathrm{dBm}$ are very noisy due to the finite number of simulated rays.

\begin{tabular}{lcccccccc}
\hline Misalignment/mm & $-\mathbf{0}$ & $\mathbf{- 0 . 5}$ & $\mathbf{- 1}$ & $\mathbf{- 1 . 5}$ & $\mathbf{- 2}$ & $\mathbf{- 2 . 5}$ & $\mathbf{- 3}$ & $\mathbf{- 3 . 5}$ \\
\hline H TX-RX: $P_{\mathrm{PD}} / \mathrm{dBm}$ & -10.1 & -8.5 & -9.5 & -12.0 & -14.1 & -15.3 & -16.0 & -26.4 \\
H TX-RX: $P_{\mathrm{PD} \text { cross } / \mathrm{dBm}}$ & -19.2 & -22.7 & -23.9 & -22.9 & -25.2 & -27.4 & -28.8 & -30.4 \\
H TX-RX: $P_{\mathrm{PD}} \mathrm{PP} / \mathrm{dBm}$ & -10.7 & -8.7 & -9.7 & -12.4 & -14.5 & -15.6 & -16.2 & -28.6 \\
S TX-RX: $P_{\mathrm{PD}} / \mathrm{dBm}$ & -13.7 & -15.2 & -20.6 & $<-40$ & $<-40$ & $<-40$ & $<-40$ & $<-40$ \\
\hline
\end{tabular}

\section{Discussion}

\subsection{TX Performance}

Figure 7 proves the suitability of the ray mapping method based on curl-free mapping for the TX part. In order to assess the performance of the design, it is meaningful to have a closer look at the loss mechanism: the main loss is represented by $\zeta_{\mathrm{TX} M}=24.7 \%$. This magnitude is quite common. It results from the extended source effect from the LD with regards to the TX lens part and the remaining curl in $\mathbf{N}$. Furthermore, the overall ray mapping performance depends on the extent of the TX output aperture and the distance to the target plane [41]. The reason for this is that ray mapping is an optical far-field design method that neglects the rays' position vector on the output aperture.

The inhomogeneity within the FOV is with $\zeta_{\mathrm{TX} H}=5.6 \%$ very low. Generally, a $\zeta_{\mathrm{TX} H}$ below $10 \%$ is a good result. The major part of the remaining inhomogeneity results from the FOV edge, where the irradiance starts to decrease. The Fresnel-loss $\zeta_{\mathrm{TX} F}$ is with $12.1 \%$ in a common region for two material interfaces. Although an anti-reflection (AR) coating might reduce this effect by a factor of 3 to 4 , it is challenging to homogeneously coat the nonplanar TX surfaces.

\subsection{Full-Channel Performance}

$P_{\mathrm{PD} P \mathrm{PP}}$ is in a sufficient range for data transfer, but how does it correspond to the data rate? For a bit error rate of $10^{-12}$ Säckinger calculates a sensitivity of $-26.5 \mathrm{dBm}$ for a $2.5 \mathrm{Gbit} / \mathrm{s}$ link and $-20.5 \mathrm{dBm}$ for a $10 \mathrm{Gbit} / \mathrm{s}$ link [26]. Tzeng et al. [54] demonstrated a sensitivity of $-21.5 \mathrm{dBm}$ for a $10 \mathrm{Gbit} / \mathrm{s}$ link. It can be concluded from Table 3 that $P_{\mathrm{PD}}$ PP is sufficient for data transmission in the Gbit/s data rate range and even provides a margin for ageing effects and other nonidealities. The optical crosstalk introduces a power penalty of up to $0.9 \mathrm{~dB}$. The magnitude of $P_{\mathrm{PD}}$ cross depends

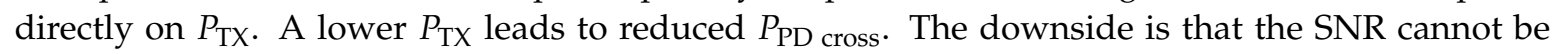
improved by increasing $P_{\mathrm{TX}}$ if the crosstalk is the dominant noise factor. The constant crosstalk part $P_{\mathrm{PD} \text { cross } 1}$ is alignment-independent. Therefore, it has to be the internal optical crosstalk. In contrast, the back-reflected signal $P_{\mathrm{PD} \text { cross } 2}$ from the opposite transceiver depends on the transceiver alignment. The crosstalk and thus the power penalty is reduced if $z$ is increased. In the far field, $P_{\mathrm{PD}}$ is decreasing with approximately $P_{\mathrm{PD}} \propto z^{-2}$ and the crosstalk scales with $P_{\mathrm{PD} \text { cross } 2} \propto z^{-4}$, since the back-reflected rays have to travel twice the distance. Although this relation is not fully correct for the near field, the trend is still valid.

The crosstalk $P_{\mathrm{PD}}$ cross 2 can be lowered by reducing the Fresnel-reflections $\zeta_{\mathrm{RX}, \mathrm{F}}$. The planar top surface is well suited for an AR coating. The problem is that the nonplanar top surface of the TX lens part is also affected by the coating. If one is able to solve the TX coating problem, a single coating is twice as effective, because the ray passes the coating two times: first at TX and second at RX. The link-budget is improved by approx. $6 \%$ per coating instead of only approx. $3 \%$. Moreover, 
the crosstalk $P_{\mathrm{PD} \text { cross } 2}$ is reduced. Alternatively, the top surface of the RX lens part could be designed nonplanar in a way that back-reflected rays miss the transmitting device.

Although TX exhibits a quite homogeneous performance, $P_{\mathrm{PD}}$ PP varies about $6.9 \mathrm{~dB}$ along radial direction for misalignment $\leq 3 \mathrm{~mm}$ according to Figure $8 \mathrm{a}$ and Table 3 . Thus, there is clearly some room for improvement for the RX lens part. The main issue results from the mismatch between near and far field in terms of ray optics. This can be seen in Figure 6a: the incident ray bundle (green) exhibits a large divergence. The situation is different at $z=50 \mathrm{~mm}$, for instance. The solid angle of incidence is much smaller and the incident ray bundle exhibits a smaller divergence. The larger the distance, the better the design approach with the edge-ray principle works. Another issue is the nonrotationally symmetric shadowing effect of the TX lens part that manifests itself as a local minimum around $(x, y)=(-2.5 \mathrm{~mm}, 0 \mathrm{~mm})$ in Figure $8 \mathrm{a}, \mathrm{c}$. The variation $\Delta P_{\mathrm{PD}}=3.29 \mathrm{~dB}$ is not crucial for data transmission. Assuming a misalignment of $-2.5 \mathrm{~mm}$ and a rotation speed of $10000 \mathrm{rpm}$, the link moves $9.42 \mu \mathrm{m}$ over the surface in Figure $8 \mathrm{a}$ during one bit-duration of $1 \mathrm{~ns}(1 \mathrm{Gbit} / \mathrm{s})$. The change of $P_{\mathrm{PD}}$ over a sequence of bits is small enough and does not influence the transmission drastically. Ideally, the receiver circuit includes a decision-point control mechanism for continuous adaptation of the decision level to improve the BER [26].

\subsection{Suitability for Rotary Scenarios}

Faulwaßer et al. [19] already reported data rates of up to $10 \mathrm{Gbit} / \mathrm{s}$. However, what data rates are generally possible compared to FORJ? From an electrical point of view, OWC is able to achieve similar data rates as single-FORJ. In contrast to FORJ, the PD has typically a larger area and thus a larger capacitance, which limits the bandwidth. Choosing a smaller PD, will reduce the maximum tolerable misalignment. The hybrid lens from this work exhibits $14.5 \mathrm{~dB}$ link loss at $(x, y)=(0 \mathrm{~mm}, 0 \mathrm{~mm})$. This link loss is a part of the active link concept. The signal is directly recovered at $\mathrm{RX}$ by amplification and optimally by subsequent analog-to-digital conversion. The magnitude of the OWC link loss depends on the magnitude of tolerable misalignment. Thus, a higher data rate requires a smaller FOV. In summary, the data rate of OWC links might be slightly below single-fiber FORJ due to a higher link loss.

With regards to the communication distance, the hybrid lens is flexible and not restricted to $z=12.5 \mathrm{~mm}$. If the lens is designed for larger ranges, the TX beam exhibits a lower divergence and $\mathrm{RX}$ should be designed for smaller angles of incidence. Thereby, the hybrid lens can be tailored to the ideal distance of the rotary system.

As mentioned in Section 2.1.3, the hybrid lens approach has the potential to be very cost effective: in contrast to fully separated TX and RX, only a single lens has to be fabricated, potentially coated and assembled.

The proposed hybrid lens works in on-axis configuration like FORJs. Future work will deal with off-axis optical links to improve flexibility. Only data rates in the range of kbit/s to a few Mbit/s have been demonstrated [2,3], which cannot compete with modern capacitive links [6]. Another interesting field of research is the realization of multi-channel optical wireless links similar to multi-FORJs. In nonrotary scenarios, data rates of several hundreds of Gbit/s have already been demonstrated [55].

\section{Conclusions}

This work has shown the suitability of OWC for full-duplex, high-speed data transfer in on-axis rotary scenarios. The proposed hybrid lens is able to provide a sufficient $\mathrm{RX}$ signal level $P_{\mathrm{PD}} \mathrm{PP}$ of more than $-16.2 \mathrm{dBm}$ even for misalignments of up to $3 \mathrm{~mm}$ at a communication distance of $z=12.5 \mathrm{~mm}$. OWC is therefore able to provide a robust data transfer without the strict mechanical tolerances compared to FORJs. The results show a maximum power penalty resulting from optical crosstalk of $0.9 \mathrm{~dB}$ within the FOV. The approach is promising since it allows low-cost fabrication. Besides the electronical components, only a single optical component is required that can be fabricated by injection molding in high volume. 


\section{Patents}

The hybrid TX-RX lens and its derivatives are covered by several patents including DE102018205559 B3 [56] (WO19197343A1 [57]). Further patents are submitted.

Author Contributions: Conceptualization, R.K., A.N.; methodology, R.K., T.S.; software, R.K.; validation, R.K. and T.S.; formal analysis, R.K.; investigation, R.K.; data curation, R.K.; writing-original draft preparation, R.K.; writing-review and editing, M.F., T.S., P.M., F.D. and A.N.; visualization, R.K.; supervision, R.K.; project administration, R.K. All authors have read and agreed to the published version of the manuscript.

Funding: This research received no external funding.

Conflicts of Interest: The authors declare no conflict of interest. The funders had no role in the design of the study; in the collection, analyses, or interpretation of data; in the writing of the manuscript, or in the decision to publish the results.

\section{Abbreviations}

The following abbreviations are used in this manuscript:

$\begin{array}{ll}\text { BER } & \text { bit error rate } \\ \text { BW } & \text { bandwidth } \\ \text { EM } & \text { emitter } \\ \text { FORJ } & \text { fiber optic rotary joint } \\ \text { FOV } & \text { field of view } \\ \text { H TX-RX } & \text { hybrid transmitter-receiver lens system } \\ \text { LED } & \text { light emitting diode } \\ \text { LD } & \text { laser diode } \\ \text { NURBS } & \text { nonuniform rational B-splines } \\ \text { OWC } & \text { optical wireless communications } \\ \text { PD } & \text { photodetector } \\ \text { RF } & \text { radio frequency } \\ \text { S TX-RX } & \text { separate transmitter-receiver lens system } \\ \text { SNR } & \text { signal-to-noise ratio } \\ \text { RX } & \text { receiver } \\ \text { TIR } & \text { total internal reflection } \\ \text { TX } & \text { transmitter }\end{array}$

\section{References}

1. Astier, R.; Capitaine, T.; Bourny, V.; Dubois, J.; Fortin, J. Technological leap of signal transfer system: CONTACTLESS. In Proceedings of the 38th Annual Conference on IEEE Industrial Electronics Society (IECON), Montreal, QC, Canada, 25-28 October 2012; pp. 3238-3243.

2. Hong, E.; Gregory, D.A.; Krishnamurthy, A. Opto-electronics based wireless slip ring system. J. Eng. Comput. Arch. 2007, 1, 1-6.

3. Wang, Z.; Gu, H.; Sun, P. Contactless signal transmission for industrial bus at rotary joint. In Proceedings of the 2015 IEEE International Conference on Signal Processing, Communications and Computing (ICSPCC), Ningbo, China, 19-22 September 2015; pp. 1-6.

4. Ghelfi, P.; Laghezza, F.; Scotti, F.; Serafino, G.; Pinna, S.; Onori, D.; Lazzeri, E.; Bogoni, A. Photonics in Radar Systems: RF Integration for State-of-the-Art Functionality. IEEE Microw. Mag. 2015, 16, 74-83. [CrossRef]

5. Nickel, H.U.; Doleschel, A.; Schmid, M. Multichannel rotary joints for surveillance radars - State-of-the-art and future trends. In Proceedings of the 14th International Radar Symposium (IRS), Dresden, Germany, 19-21 June 2013; pp. 282-287.

6. Nickel, H.U.; Zovo, J.; Schmid, M. Refining Radar Architectures: Multichannel Rotary Joints for Surveillance Radars. IEEE Microw. Mag. 2016, 17, 60-74. [CrossRef]

7. Vollbracht, D.; Gekat, F.; Hilger, D.; Hille, M. Waveguide fibre optic rotary joint for antenna mounted radar receivers. In Proceedings of the 34th Conference on Radar Meteorology, Poster, Williamsburg, VA, USA, 5-9 October 2009. 
8. Mi, L.; Yao, S.-L.; Sun, C.-D.; Sun, B.; Zhang, H.-J. A single-channel fiber optic rotary joint on the basis Of TEC fiber collimators. In Proceedings of the International Conference on Optical Communications and Networks (ICOCN 2010), Nanjing, China, 24-27 October 2010; pp. 437-440.

9. Roberts, G.; Hadfield, P.; Humphries, M.E.; Bauder, F.; Izquierdo, J.M.G. Design and evaluation of the power and data contactless transfer device. In Proceedings of the 1997 IEEE Aerospace Conference, Snowmass, Aspen, CO, USA, 10 Febuary 1996; Volume 3, pp. 523-533.

10. Song, Y.; Wang, S.; Feng, Y. Stable and Secure Contactless Connectivity of Power and Data in Rotational Applications. In Proceedings of the 2018 IEEE Asia-Pacific Conference on Antennas and Propagation (APCAP), Auckland, New Zealand, 5-8 August 2018; pp. 401-402.

11. Patyuchenko, A. 60 GHz Wireless Data Interconnect for Slip Ring Applications; Technical Report; Analog Devices, Inc: Norwood, MA, USA, 2019.

12. Timsit, R.S.; Antler, M. Tribology of Electronic Connectors: Contact Sliding Wear, Fretting, and Lubrication: 7.4. Lubrication. In Electrical Contacts: Principles and Applications; Slade, P.-G., Ed.; CRC Press: Boca Raton, FL, USA, 2014; pp. 481-506.

13. Doleschel, A.; Lege, M. Contactless solutions for radar rotary joint systems. In Proceedings of the 16 th International Radar Symposium (IRS), Dresden, Germany, 24-26 June 2015; pp. 451-456.

14. Panhans, C.; Stolle, R. High Bandwidth Contactless Rotary Transmitter Design Optimized for Baseband Transmission. In Proceedings of the 11th International Conference on Information Technology and Electrical Engineering (ICITEE), Pattaya, Thailand, 10-11 October 2019; pp. 1-6.

15. Wageningen, D.V.; Staring, T. The Qi wireless power standard. In Proceedings of the 14th International Power Electronics and Motion Control Conference (EPE-PEMC), Ohrid, Macedonia, 6-8 September 2010; pp. S15-25-S15-32.

16. Dorsey, G.F.; Coleman, D.S.; Witherspoon, B.K. High Speed Data across Sliding Electrical Contacts. In Proceedings of the 2012 IEEE 58th Holm Conference on Electrical Contacts (Holm), Portland, OR, USA, 23-26 September 2012; pp. 1-12.

17. Chen, H; Hsu, S.; Chang, T.; Chi, W.; Ma, S.; Wu, T. Signal integrity design for GHz high-speed differential wires with slip ring capsule. In Proceedings of the 2012 IEEE International Symposium on Electromagnetic Compatibility, Pittsburgh, PA, USA, 6-10 August 2012; pp. 18-21.

18. Slade, P.-G. Electrical Contacts: Principles and Applications; CRC Press: Boca Raton, FL, USA, 2014.

19. Faulwaßer, M.; Kirrbach, R.; Schneider,T.; Noack, A. 10 Gbit/s bidirectional transceiver with monolithic optic for rotary connector replacements. In Proceedings of the 2018 Global LIFI Congress (GLC), Paris, France, 8-9 February 2018; pp. 95-102.

20. Rappaport, T,S.; Sun, S.; Mayzus, R.; Zhao, H.; Azar, Y.; Wang, K.; Wong, G. N.; Schulz, J.K.; Samimi, M.; Gutierrez, F. Millimeter Wave Mobile Communications for 5G Cellular: It Will Work!. IEEE Access 2013, 1, 335-349. [CrossRef]

21. Aizawa, T.; Sakai, M.; Toguchi, S.; Hirohashi, K. Optical Slip Rings for Home Security High-Definition Cameras. In Proceedings of the 2007 Digest of Technical Papers International Conference on Consumer Electronics, Las Vegas, NV, USA, 10-14 January 2007; pp. 1-2.

22. Guenter, J.K.; Hawthorne, R.A.; Granville, D.N.; Hibbs-Brenner, M.K.; Morgan, R.A. Reliability of proton-implanted VCSELs for data communications.In Proceedings of the SPIE 2683, Fabrication, Testing, and Reliability of Semiconductor Lasers, San Jose, CA, USA, 4 April 1996; pp. 102-113.

23. Christou, A.; Unger, B.A. Semiconductor Device Reliability; Christou, A., Unger, B.A., Eds.; Springer: Dordrecht, The Netherlands, 1990; p. 60.

24. Vinh, Q.T.; Khanh, T.Q.; Ganev, H.; Wagner, M. Measurement and Modeling of the LED Light Source. In LED Lighting: Technology and Perception, 1st ed.; Khanh, T.Q., Bodrogi, P., Vinh, Q.T., Winkler, H., Eds.; John Wiley \& Sons, Ltd: Hoboken, NJ, USA, 2014; pp. 214-222.

25. Dorsey, G.F. Fiber Optic Rotary Joints - A Review. IEEE Trans. Compon. Hybrids Manuf. Technol. 1982, 5, 37-41. [CrossRef]

26. Säckinger, E. Analysis and Design of Transimpedance Amplifiers for Optical Receivers, 1st ed.; John Wiley and Sons: Hoboken, NJ, USA, 2018; pp. 46, 48, 56, 120-127, 197-199, 469-474.

27. Safety of Laser Products - Part 1: Equipment Classification and Requirements; IEC 60825-1:2014; IEC: Geneva, Switzerland, 2014. 
28. Kirrbach, R.; Jakob, B.; Noack, A. Introducing Advanced Freeform Optic Design to Li-Fi Technology. In Proceedings of the 7th International Conference on Photonics, Optics and Laser Technology (PHOTOPTICS 2019), Prague, Czech Republic, 25-27 February 2019; pp. 249-250.

29. Kahn, J.; Barry, J.R. Wireless Infrared Communications. Proc. IEEE 1997, 85, 265-298. [CrossRef]

30. Welford, W.T.; Winston, R. High Collection Nonimaging Optics, 1st ed.; Academic Press Inc.: San Diego, CA, USA, 1989; pp. 1-28, 54.

31. Kirrbach, R.; Faulwaßer, M.; Jakob, B. Non-rotationally Symmetric Freeform Fresnel-Lenses for Arbitrary Shaped Li-Fi Communication Channels. In Proceedings of the 2019 Global LIFI Congress (GLC), Paris, France, 12-13 June 2019; pp. 103-108.

32. Stepniak, G.; Kowalczyk, M.; Maksymiuk, L.; Siuzdak, J. Transmission Beyond 100 Mbit/s Using LED Both as a Transmitter and Receiver. IEEE Photonics Technol. Lett. 2015, 27, 2067-2070. [CrossRef]

33. Serial Infrared Transceiver (SIR), $115.2 \mathrm{kbit} / \mathrm{s}, 2.4 \mathrm{~V}$ to $5.5 \mathrm{~V}$ Operation TFBS4711, Vishay Semiconductors, rev. 3.3, 08.04.2019. Available online: http:/ / www.vishay.com (accessed on 30 November 2019).

34. Faulwaßer, M.; Deicke, F.; Schneider, T. 10 Gbit/s bidirectional optical wireless communication module for docking devices. In Proceedings of the 2014 IEEE Globecom Workshops (GC Wkshps), Austin, TX, USA, 8-12 December 2014; pp. 512-517.

35. Manousiadis, P.; Rajbhandari, S.; Mulyawan, R.; Vithanage, D.; Chun, H.; Faulkner, G.; O’brien, D.; Turnbull, G.; Collins, S.; Samuel,I. Wide field-of-view fluorescent antenna for visible light communications beyond the étendue limit. Optica 2016, 3, 702-706. [CrossRef]

36. Naumann, H.; Schröder, G.; Löffler-Mang, M. Handbuch Bauelemente der Optik: Grundlagen, Werkstoffe, Geräte, Messtechnik, 7th ed.; Carl Hanser Verlag: Munich Vienna, Germany, 2014; pp. 69-70, 81-83.

37. Mäkinen, J.-K. Cost Modeling of Injection-Molded Plastic Optics. In OECC Handbook of Plastic Optics, 2nd ed.; Bäumer, S., Ed.; WILEY-VCH Verlag GmbH and Co. KGaA: Weinheim, Germany, 2010; p. 229.

38. Miñano, J.C. SMS 3D Design Method. In Illumination Engineering: Design of Nonimaging Optics; Anderson, J., Ed.; Wiley-IEEE Press: Hoboken, NJ, USA, 2013; p. 102.

39. Wang, K.; Liu, S.; Xiaobing, L.; Wu, D. Basic Algorithms of Freeform Optics for LED Lighting. In Freeform Optics for LED Packages and Applications, 1st ed.; John Wiley and Sons: Singapore, 2017; pp. 25-69.

40. Ma, D. Exploration of Ray Mapping Methodology in Freeform Optics. Ph.D. Thesis, The University of Arizona, Tucson, AZ, USA, 2015.

41. Bruneton, A.; Bäuerle, A; Wester, R.; Stollenwerk, J.; Loosen, P. Limitations of the ray mapping approach in freeform optics design. Opt. Lett. 2013, 38, 1945-1947. [CrossRef]

42. Desnijder, K.; Hanslaer, P.; Meuret, Y. Flexible design method for freeform lenses with an arbitrary lens contour. Opt. Lett. 2017, 42, 5238-5241. [CrossRef]

43. Desnijder, K.; Hanslaer, P.; Meuret, Y. A ray mapping method for off-axis and non-paraxial freeform illumination lens design. Opt. Lett. 2019, 44, 771-774. [CrossRef] [PubMed]

44. Desnijder, K.; Ryckaert, W; Hanslaer, P.; Meuret, Y. Luminance spreading freeform lens arrays with accurate intensity control. Opt. Express 2019, 27, 32994-33004. [CrossRef] [PubMed]

45. Wu, R.; Liu, P.; Zhang, Y.; Zheng, Z.; Li, H.; Liu, X. A mathematical model of the single freeform surface design for collimated beam shaping. Opt. Express 2013, 21, 20976-20989. [CrossRef] [PubMed]

46. Wu, R.; Zhang, Y; Sulman, M.M.; Zheng, Z.; Benítez, P.; Miñano, J.C. Initial design with L2 Monge-Kantorovich theory for the Monge-Ampère equation method in freeform surface illumination design. Opt. Express 2014, 22, 16161-16177. [CrossRef]

47. Michaelis, D.; Schreiber, P.; Bräuer, A. Cartesian oval representation of freeform optics in illumination systems. Opt. Lett. 2011, 36, 918-920. [CrossRef]

48. Winston, R.; Minano, J.C.; Benitez, P.G.; Shatz, N.; Bortz, J.C. Nonimaging Optics, 1st ed.; Academic Press: Burlington, MA, USA, 2005; pp. 181-218.

49. Benitez, P.G.; Miñano, J.C.; Blen, J.; Arroyo, R.M.; Chaves, J.; Dross, O.; Hernandez, M.; Falicoff, W. Simultaneous multiple surface optical design method in three dimensions. Opt. Eng. 2004, 43, 1489-1502. [CrossRef]

50. Ries, H.; Muschaweck, J. Tailored freeform optical surfaces. J. Opt. Soc. Am. 2002, 19, 590-595. [CrossRef]

51. Wallhead, I.; Jiménez, T.M.; Ortiz, J.V.G.; Toledo, I.G.; Toledo, C.G. Design of an efficient Fresnel-type lens utilizing double total internal reflection for solar energy collection. Opt. Express 2010, 20, A1005-A1010. [CrossRef] 
52. Piegl, L.; Tiller, W.J. The NURBS Book, 2nd ed.; Springer: Berlin/Heidelberg, Germany, 1995.

53. Ning, X.; Winston, R.; O'Gallagher, J. Dielectric totally internally reflecting concentrators. Appl. Opt. 1987, 26, 300-305. [CrossRef]

54. Tzeng, L.D.; Mizuhzra, O.; Nguyen, T.V.; Ogawa, K.; Watanabe, I.; Makita, K.; Tsuji, M.; Taguchi, K. A high-sensitivity APD receiver for 10-Gb/s system applications. IEEE Photonics Technol. Lett. 1996, 8, 1229-1231. [CrossRef]

55. Gomez, A.; Shi, K.; Quintana, C.; Maher, R.; Faulkner, G.; Bayvel, P.; Thomsen, B.C.; O’Brien, D. Design and Demonstration of a 400 Gb/s Indoor Optical Wireless Communications Link. J. Lightw. Technol. 2016, 34, 5332-5339. [CrossRef]

56. Schneider, T. Optische Sende-/Empfangs-Einheit und Vorrichtung zur Signalübertragung. DE102018205559 B3, 12 April 2018.

57. Schneider, T. Optical Transceiver Unit and Device for Signal Transmission. Patent No. WO19197343A1, 8 April 2019.

(C) 2020 by the authors. Licensee MDPI, Basel, Switzerland. This article is an open access article distributed under the terms and conditions of the Creative Commons Attribution (CC BY) license (http:/ / creativecommons.org/licenses/by/4.0/). 